PROCEEDINGS OF THE

AMERICAN MATHEMATICAL SOCIETY

Volume 126, Number 4, April 1998, Pages 1017-1022

S 0002-9939(98)04160-4

\title{
REMARKS ON THE NON-COHEN-MACAULAY LOCUS OF NOETHERIAN SCHEMES
}

\author{
NGUYEN TU CUONG
}

(Communicated by Wolmer V. Vasconcelos)

\begin{abstract}
In this paper we give a notion of polynomial type $p(X)$ of a Noetherian scheme $X$ and define the function $d p: X \longrightarrow \mathbb{Z}$ by $d p(x)=$ $\operatorname{dim} O_{X, x}-p\left(O_{X, x}\right)$ for all $x \in X$. Then we show that if $X$ admits a dualizing complex and $X$ is equidimensional, $d p$ is (lower) semicontinuous; moreover, in that case, the non-Cohen-Macaulay locus $\operatorname{nCM}(X)=\left\{x \in X \mid O_{X, x}\right.$ is not Cohen-Macaulay $\}$ is biequidimensional iff $d p$ is constant on $\operatorname{nCM}(X)$.
\end{abstract}

\section{INTRODUCTION}

Let $X$ be a noetherian scheme with the structure sheaf $O_{X}$. The non-CohenMacaulay locus $\operatorname{nCM}(X)$ of $X$ is defined by $\operatorname{nCM}(X)=\left\{x \in X \mid O_{X, x}\right.$ is not a Cohen-Macaulay ring $\}$. It is well-known that if $X$ admits a dualizing complex then $\operatorname{nCM}(X)$ is a closed subscheme of $X([\mathrm{H} 1])$. Then, we say that $\mathrm{nCM}(X)$ is biequidimensional if it is equidimensional and equicodimensional, i.e. all irreducible components of $\operatorname{nCM}(X)$ have the same dimension and codimension. Therefore it is natural to ask following questions:

(i) How can one estimate the dimension of $\operatorname{nCM}(X)$ ?

(ii) When is $\mathrm{nCM}(X)$ biequidimensional?

The purpose of this paper is to deal with these questions. First, for the local scheme $X=\operatorname{Spec} A$, where $A$ is a local ring, the question (i) has been studied in $[\mathrm{C} 1]$ and [C2]. There we defined the polynomial type of a local ring $A$ as follows. Let $\underline{x}$ be a system of parameters and $\underline{n}=\left(n_{1}, \ldots, n_{d}\right)$ a d-tuple of positive integers. Then the least degree of all polynomials in $\underline{n}$ bounding the function

$$
I(\underline{n} ; \underline{x})=\ell\left(A /\left(x_{1}^{n_{1}}, \ldots, x_{d}^{n_{d}}\right) A\right)-n_{1} \ldots n_{d} e(\underline{x} ; A)
$$

from above (where $\ell(N)$ is the length of the $A$-module $N$ and $e(\underline{x} ; A)$ is the Serre multiplicity of $A$ with respect to $\underline{x}$ ) is independent of the choice of the system of parameters $\underline{x}$. This invariant is called the polynomial type of $A$ and denoted by $p(A)$ (see $[\mathrm{C} 2]$ for details). We will extend this notion to schemes and show in Section 2 (Theorem 2.4) that the polynomial type of the scheme $X$ is equal to dim $\operatorname{nCM}(X)$ provided $X$ is equidimensional and admits a dualizing complex. Second, relative to the polynomial type we can define on $X$ a function $d p: X \longrightarrow \mathbb{Z}$ by $d p(x)=\operatorname{dim} O_{X, x}-p\left(O_{X, x}\right)$ for all $x \in X$, where $\mathbb{Z}$ is the set of all integers. Then

Received by the editors July 3, 1995 and, in revised form, October 7, 1996.

1991 Mathematics Subject Classification. Primary 13C99; Secondary 13H10, 14M99.

The author is partially supported by the National Basic Research Program of Vietnam.

(C) 1998 American Mathematical Society 
we will prove in Section 3, under the same assumptions for $X$ as above, that this function is (lower) semicontinuous (Theorem 3.2). As a consequence of this result we get a criterion for the biequidimensionality of $\mathrm{nCM}(X)$ which says that $\mathrm{nCM}(X)$ is biequidimensional if and only if $d p$ is constant on $\operatorname{nCM}(X)$ (Theorem 3.4).

The reader may consult [G], [H1] and [H2] for unexplained terminology and notations.

\section{The Polynomial type of a SChEme}

First, we recall the definition of polynomial type of a local ring $A$ given in [C2]. Let $\underline{x}=\left(x_{1}, \ldots, x_{d}\right)$ be a system of parameters of $A$ and $\underline{n}=\left(n_{1}, \ldots, n_{d}\right)$ a $d$ tuple of positive integers. We consider the difference between the length and the multiplicity

$$
I(\underline{n} ; \underline{x})=\ell\left(A /\left(x_{1}^{n_{1}}, \ldots, x_{d}^{n_{d}}\right) A\right)-n_{1} \ldots n_{d} e(\underline{x} ; A)
$$

as a function in $\underline{n}$. Then the least degree of all polynomials bounding $I(\underline{n} ; \underline{x})$ from above is independent of the choice of $\underline{x}$. This numerical invariant is called the polynomial type of $A$ and denoted by $p(A)$. For further use in this paper, we need the following remarks and results from $[\mathrm{C} 1]$ and $[\mathrm{C} 2]$ about the polynomial type of a local ring $A$.

(2.1) If $A$ is a Cohen-Macaulay ring, we set $p(A)=-\infty$. Then, if $A$ is not CohenMacaulay, $0 \leq p(A) \leq \operatorname{dim} A-1$.

(2.2) Denote by $\mathfrak{a}_{i}(A)$ the annihilator of the $i$-th local cohomology module $H_{m}^{i}(A)$ of $A$ with respect to the maximal ideal $\mathfrak{m}$. Put $\mathfrak{a}(A)=\mathfrak{a}_{0}(A) \ldots \mathfrak{a}_{d-1}(A)$, where $d=\operatorname{dim} A$. Suppose that $A$ admits a dualizing complex. Then by [H1], the nonCohen-Macaulay locus $\operatorname{nCM}(A)$ of $A$ is a closed set in $\operatorname{Spec} A$. Then the following two assertions are true (see [C1, Theorem 1.2] and [C2, Theorem 3.3]):

(i) $\operatorname{dim} \operatorname{nCM}(A) \leq p(A)=\operatorname{dim} A / \mathfrak{a}(A)$.

(ii) If $A$ is equidimensional then $\operatorname{dim} \operatorname{nCM}(A)=p(A)$.

(2.3) With the same method that we used in the proof of Theorem $4.1 \mathrm{in}[\mathrm{C} 2]$, we can show the following result: Suppose that $A$ admits a dualizing complex and $k$ is a positive integer. Then the following conditions are equivalent:

(i) $p(A) \leq k$.

(ii) For any $\mathfrak{p} \in \operatorname{Spec} A$ with $\operatorname{dim} A / \mathfrak{p}>k, A_{\mathfrak{p}}$ is Cohen-Macaulay and $\operatorname{dim} A_{\mathfrak{p}}+$ $\operatorname{dim} A / \mathfrak{p}=\operatorname{dim} A$.

(2.4) (see $\left[\mathrm{C} 2\right.$, Corollary 5.3]). Let $\mathfrak{p}$ be a prime ideal of $A$. Then $p(A) \geq p\left(A_{\mathfrak{p}}\right)+$ $\operatorname{dim} A / \mathfrak{p}$.

Now we want to extend the notion of polynomial type to a noetherian scheme.

(2.5) Definition. Let $X$ be a noetherian scheme. We set $p(x)=p\left(O_{X, x}\right)$ for any $x \in X$ and define the polynomial type of $X$ by

$$
p(X)=\sup _{x \in X} p(x) .
$$

(2.6) Remark. As in the local case, we have $-\infty \leq p(X) \leq \operatorname{dim} X-1$, and $X$ is a Cohen-Macaulay scheme (i.e. $O_{X, x}$ is Cohen-Macaulay for all $x \in X$ ) iff $p(X)=-\infty$. Therefore $p(x) \geq 0$ if and only if $x \in \operatorname{nCM}(X)$.

Denote by $F$ the set of all closed points of $X$. Then we have an easy lemma. 
(2.7) Lemma. Let $X, F$ be as above. Then

$$
p(X)=\sup _{x \in F} p(x) .
$$

Proof. It is enough to prove this for the affine scheme $X=\operatorname{Spec} A$, where $A$ is a noetherian ring. Let $x \in X$ be an arbitrary point corresponding to a prime ideal $\mathfrak{p} \subseteq A$. Then there exists a maximal ideal $\mathfrak{m}$ of $A$ such that $\mathfrak{p} \subseteq \mathfrak{m}$. Hence, by (2.4), $p\left(A_{\mathfrak{p}}\right) \leq p\left(A_{\mathfrak{m}}\right)-\operatorname{dim}\left(A_{\mathfrak{m}} / \mathfrak{p} A_{\mathfrak{m}}\right) \leq p\left(A_{\mathfrak{m}}\right)$. Therefore $p(X) \leq \sup _{x \in F} p\left(O_{X, x}\right)$.

Similarly to the local case, we have the following theorem which gives a geometric meaning of the polynomial type of a scheme.

(2.8) Theorem. Let $X$ be a noetherian scheme admitting a dualizing complex. Then

(i) $p(X) \geq \operatorname{dim} \operatorname{nCM}(X)$.

(ii) If $X$ is locally equidimensional, then the equality holds in (i).

Proof. Note first that since $X$ admits a dualizing complex, $X$ is of finite dimension and $\operatorname{nCM}(X)$ is a closed subscheme of $X$ by [H1]. We only need to prove our claim for the affine scheme $X=\operatorname{Spec} A$.

(i). Let $\mathfrak{p}$ be a prime ideal of $A$ contained in $\operatorname{nCM}(A)$ such that $\operatorname{dim} A / \mathfrak{p}=$ $\operatorname{dim} \operatorname{nCM}(A)$. Let $\mathfrak{m}$ be a maximal ideal of $A$ such that $\mathfrak{p} \subseteq \mathfrak{m}$ and $\operatorname{dim}\left(A_{\mathfrak{m}} / \mathfrak{p} A_{\mathfrak{m}}\right)=$ $\operatorname{dim}(A / \mathfrak{p})$. As $\mathfrak{p} A_{\mathfrak{m}} \in \operatorname{nCM}\left(A_{\mathfrak{m}}\right)$, we get $\operatorname{dim} \mathrm{nCM}\left(A_{\mathfrak{m}}\right) \geq \operatorname{dim} \mathrm{nCM}(A)$. The converse inequality $\operatorname{dim} \operatorname{nCM}\left(A_{\mathfrak{n}}\right) \leq \operatorname{dim} \operatorname{nCM}(A)$ is easily shown to hold true for any maximal ideal $\mathfrak{n}$ of $A$. So we get $\operatorname{dim} \operatorname{nCM}\left(A_{\mathfrak{m}}\right)=\operatorname{dim} \operatorname{nCM}(A)$. Hence by $(2.2)$, (i) we get $p(A) \geq p\left(A_{\mathfrak{m}}\right) \geq \operatorname{dimnCM}\left(A_{\mathfrak{m}}\right)=\operatorname{dimnCM}(A)$.

(ii). Now, by (2.7) we may assume that there exists a maximal ideal $\mathfrak{n}$ of $A$ such that $p(A)=p\left(A_{\mathfrak{n}}\right)$. Then by $(2.2)$, (ii) we get $p(A)=p\left(A_{\mathfrak{n}}\right)=\operatorname{dimnCM}\left(A_{\mathfrak{n}}\right) \leq$ $\operatorname{dim} n C M(A)$. Thus $p(A)=\operatorname{dim} n C M(A)$, as required.

(2.9) Example. This example shows that the equality $p(X)=\operatorname{dim} n \mathrm{nM}(X)$ does not hold without the assumption that $X$ is equidimensional. For $d \geq 2$, we set $A=$ $k\left[X_{1}, \ldots, X_{d}, Y\right] /\left(X_{1} Y, \ldots, X_{d} Y\right)$, where $X_{1}, \ldots, X_{d}$ and $Y$ are indeterminates over an algebraically closed field $k$. Then $\operatorname{dim} A=d$. It is easy to check that $\mathrm{nCM}(A)=$ $\{\mathfrak{m}\}$, where $\mathfrak{m}=\left(X_{1}, \ldots, X_{d}, Y\right) A$; thus $\operatorname{dim} \operatorname{nCM}(A)=0$ and $p(A)=p\left(A_{\mathfrak{m}}\right)$. Let $\underline{y}=\left(y_{1}, \ldots, y_{d}\right)$ be a system of parameters of $A_{\mathfrak{m}}$ defined by $y_{i}=X_{i} A_{\mathfrak{m}}$ for $i=1, \ldots, d-1$ and $y_{d}=\left(X_{d}+Y\right) A_{\mathfrak{m}}$. Then we can show in $A_{\mathfrak{m}}$ that $I(\underline{n} ; \underline{y})=n_{d}+1$ is a polynomial of degree 1 . Therefore $p(A)=1$.

\section{Biequidimensionality of $\mathrm{nCM}(\mathrm{X})$}

We start with the following fact.

(3.1) Lemma. Let $X$ be a noetherian scheme of finite dimension. Then for all $x \in X$ we have

(i) $p(X) \geq p(x)+\operatorname{dim} \overline{\{x\}}$.

(ii) If $X$ is locally equidimensional and admits a dualizing complex, then $\operatorname{dim} O_{X, x}$ $-p(x) \geq \operatorname{dim} O_{X, y}-p(y)$ for any specialization $y$ of $x$.

Proof. For $x \notin n \operatorname{nCM}(X)$, there is nothing to prove. Thus we may assume that $x \in \operatorname{nCM}(X)$.

(i). Let $y$ be an arbitrary point in $\overline{\{x\}}$. By (2.4) we have $p(X) \geq p(y) \geq$ $p(x)+\operatorname{dim} O_{\overline{\{x\}}, y}$. Therefore $p(X) \geq p(x)+\operatorname{dim} \overline{\{x\}}$. 
(ii). It follows from the proof of (i) that

$$
\operatorname{dim} O_{X, x}-p(x) \geq \operatorname{dim} O_{X, x}+\operatorname{dim} O_{\overline{\{x\}}, y}-p(y) .
$$

Since $X$ admits a dualizing complex, $X$ is catenary by [H1]. Hence, we deduce from the local equidimensionality of $X$ that $\operatorname{dim} O_{\overline{\{x\}}, y}=\operatorname{dim} O_{X, y}-\operatorname{dim} O_{X, x}$. Therefore $\operatorname{dim} O_{X, x}-p(x) \geq \operatorname{dim} O_{X, y}-p(y)$, as required.

Now let $\mathbb{Z}$ be the set of all integers. We consider the function $p: X \longrightarrow \mathbb{Z}$ defined by $p(x)=p\left(O_{X, x}\right)$ for all $x \in X$. Then Lemma 3.1, (i) leads us to the following question: is the function $p$ semicontinuous? Unfortunately, it is not true in general. But, with the same method as used in [C-M], we can prove that if $X$ admits a dualizing complex then the function $p$ is (upper) semicontinuous if and only if $p(X) \leq 1$. On the other hand, (ii) of Lemma 3.1 leads us to study the function $d p: X \longrightarrow \mathbb{Z}$ defined by $d p(x)=\operatorname{dim} O_{X, x}-p(x)$ for all $x \in X$. We have the following theorem.

(3.2) Theorem. Let $X$ be a noetherian scheme admitting a dualizing complex. Suppose that $X$ is locally equidimensional. Then the function $d p: X \longrightarrow \mathbb{Z}$ is (lower) semicontinuous.

To prove this theorem we need the following lemma.

(3.3) Lemma. Let $X$ be as in Theorem 3.2. Let $x \in \operatorname{nCM}(X)$. Set $V_{x}=\{z \mid z$ is a generic point of $\mathrm{nCM}(X)$ such that $x \in \overline{\{z\}}\}$. Then

$$
d p(x)=\min _{z \in V_{x}} d p(z) .
$$

Proof. It is enough to prove the lemma in case $X$ is an affine scheme. Suppose that $X=\operatorname{Spec} A$. Let $\mathfrak{p}$ be a prime ideal of $A$ corresponding to $x$. Then

$$
V_{x}=\{\mathfrak{q} \in \operatorname{nCM}(A) \mid \mathfrak{q} \subseteq \mathfrak{p} \text { and } \mathfrak{q} \text { is minimal in } \operatorname{nCM}(A)\} .
$$

Let $\mathfrak{q}_{o} \in V_{x}$ be such that $d p\left(\mathfrak{q}_{o}\right)=\min _{\mathfrak{q} \in V_{x}} d p(\mathfrak{q})$. Since $d p(\mathfrak{p}) \leq d p\left(\mathfrak{q}_{o}\right)$ by (3.1), (ii), it remains to show that $d p(\mathfrak{p}) \geq d p\left(\mathfrak{q}_{o}\right)$. Note that by the choice of $\mathfrak{q}_{o}$, if $\mathfrak{q}^{\prime}$ is a prime ideal properly contained in $\mathfrak{q}_{o}$ then $\mathfrak{q}^{\prime} \notin \operatorname{nCM}(A)$. Morerover, since $A$ is equidimensional and catenary, it follows by $(2.1)$ and $(2.3)$ that $p\left(A_{\mathfrak{q}_{o}}\right)=0$. Therefore, if we can prove that $\operatorname{dim}\left(A / \mathfrak{q}_{o}\right)_{\mathfrak{p}} \geq p\left(A_{\mathfrak{p}}\right)$ then

$$
\operatorname{dim} A_{\mathfrak{p}}-\operatorname{dim} A_{\mathfrak{q}_{o}}=\operatorname{dim}\left(A / \mathfrak{q}_{o}\right)_{\mathfrak{p}} \geq p\left(A_{\mathfrak{p}}\right),
$$

and the above inequality follows. On the other hand, by virtue of (2.3), to prove that $\operatorname{dim}\left(A / \mathfrak{q}_{o}\right)_{\mathfrak{p}} \geq p\left(A_{\mathfrak{p}}\right)$ we only have to show that if $\mathfrak{q}$ is a prime ideal contained in $\mathfrak{p}$ with $\operatorname{dim}(A / \mathfrak{q})_{\mathfrak{p}}>\operatorname{dim}\left(A / \mathfrak{q}_{o}\right)_{\mathfrak{p}}$ then $\mathfrak{q} \notin \mathrm{nCM}(A)$. In fact, suppose that there is a prime ideal $\mathfrak{q} \in \mathrm{nCM}(X)$ contained in $\mathfrak{p}$ with $\operatorname{dim}(A / \mathfrak{q})_{\mathfrak{p}}>\operatorname{dim}\left(A / \mathfrak{q}_{o}\right)_{\mathfrak{p}}$. Without loss of generality we may assume that $\mathfrak{q} \in V_{x}$. Therefore $\operatorname{dim} A_{\mathfrak{q}}-p\left(A_{\mathfrak{q}}\right) \geq \operatorname{dim} A_{\mathfrak{q}_{o}}$. This implies that

$$
\operatorname{dim} A_{\mathfrak{p}}-\operatorname{dim} A_{\mathfrak{q}}+p\left(A_{\mathfrak{q}}\right) \leq \operatorname{dim} A_{\mathfrak{p}}-\operatorname{dim} A_{\mathfrak{q}_{o}}=\operatorname{dim}\left(A / \mathfrak{q}_{o}\right)_{\mathfrak{p}}
$$

thus, since $\operatorname{dim}\left(A / \mathfrak{q}_{o}\right)_{\mathfrak{p}}<\operatorname{dim}(A / \mathfrak{q})_{\mathfrak{p}}=\operatorname{dim} A_{\mathfrak{p}}-\operatorname{dim} A_{\mathfrak{q}}$,

$$
\operatorname{dim}\left(A / \mathfrak{q}_{o}\right)_{\mathfrak{p}}+p\left(A_{\mathfrak{q}}\right)<\operatorname{dim}\left(A / \mathfrak{q}_{o}\right)_{\mathfrak{p}}
$$

This contradicts the inequality $p\left(A_{\mathfrak{q}}\right) \geq 0$. The lemma is proved.

We are now ready to prove the theorem. 
Proof of Theorem (3.2). As in the proof of Lemma 3.3, it is enough to show our claim for the affine case $X=\operatorname{Spec} A$. We have to prove that for each non-negative integer $t$ the set $U_{t}=\{\mathfrak{p} \in A \mid d p(\mathfrak{p}) \geq t\}$ is an open set. By (3.1), (ii) $U_{t}$ is stable under generalization. Therefore, by $[\mathrm{M}],(22 \mathrm{~B})$ we only need to prove that if $\mathfrak{p} \in U_{t}$ then $U_{t}$ contains a non-empty open set of the irreducible closed set $V(\mathfrak{p})$. Since $\mathrm{nCM}(A)$ is closed, there exists a finite set of prime ideals $W=\left\{\mathfrak{p}_{1}, \ldots, \mathfrak{p}_{n}\right\}$ such that $V\left(\mathfrak{p}_{1}\right), \ldots, V\left(\mathfrak{p}_{n}\right)$ are the different irreducible components of $\mathrm{nCM}(A)$. We set $V=\left\{\mathfrak{p}_{i} \in W \mid \mathfrak{p}_{i} \not \mathbb{p}\right\}$ and $P=\bigcap_{\mathfrak{p}_{i} \in V} \mathfrak{p}_{i}$. Then there always exists an element $f \in P \backslash \mathfrak{p}$. Let $Y_{f}=\operatorname{Spec}(A / \mathfrak{p})_{f}$. We will show that for all $\mathfrak{q} \in Y_{f}, d p(\mathfrak{q}) \geq t$. In fact, by Lemma $(3.3)$ we get $d p(\mathfrak{q})=\min _{\substack{\mathfrak{p}_{i} \in W \\ \mathfrak{p}_{i} \subseteq \mathfrak{q}}} d p\left(\mathfrak{p}_{i}\right)$ and $d p(\mathfrak{p})=\min _{\mathfrak{p}_{i} \in W \backslash V} d p\left(\mathfrak{p}_{i}\right)$. But, it is easy to see by the choice of $f$ that $W \backslash V=\left\{\mathfrak{p}_{i} \in W \mid \mathfrak{p}_{i} \subseteq \mathfrak{q}\right\}$. Thus $d p(\mathfrak{q})=d p(\mathfrak{p}) \geq t$, and the proof of Theorem (3.2) is complete.

Theorem (3.2) leads to a criterion for the biequidimensionality of $\operatorname{nCM}(X)$ as follows. Recall that $\operatorname{nCM}(X)$ is said to be biequidimensional if it is equidimensional and equicodimensional, i.e. all irreducible components of $\operatorname{nCM}(X)$ have the same dimension and codimension.

(3.4) Theorem. Let $X$ be as in Theorem (3.2). Then the following conditions are equivalent:

(i) $\mathrm{nCM}(X)$ is a biequidimensional scheme.

(ii) $\left.d p\right|_{\operatorname{nCM}(X)}: \operatorname{nCM}(X) \longrightarrow \mathbb{Z}$ is a constant function.

Proof. (i) $\Rightarrow$ (ii). Let $Z$ be an arbitrary irreducible component of $\operatorname{nCM}(X)$ with the unique generic point $z$. Since $X$ is locally equidimensional and catenary, $p(z)=0$ by $(2.1)$ and (2.3). Therefore $d p(z)=\operatorname{codim} \operatorname{nCM}(X)$, since $\mathrm{nCM}(X)$ is equicodimensional. Thus, we may assume that $X=\operatorname{Spec} A$, where $A$ is an equidimensional ring which admits a dualizing complex. We have to prove that $d p(\mathfrak{p})=d p(\mathfrak{q})$ for two arbitrary prime ideals $\mathfrak{p} \subset \mathfrak{q}$ contained in $\operatorname{nCM}(X)$. Now, by localizing at $\mathfrak{q}$, we may assume without any loss of generality that $A$ is a local ring with the maximal ideal $\mathfrak{q}$. Since $d p(\mathfrak{p}) \geq d p(\mathfrak{q})$ by $(3.1)$, (ii), it remains to show that $d p(\mathfrak{q}) \geq d p(\mathfrak{p})$. This is equivalent to proving that $p\left(A_{\mathfrak{p}}\right) \geq p(A)-\operatorname{dim} A / \mathfrak{p}$. But, applying (2.3) to the ring $A_{\mathfrak{p}}$, we only have to verify that there exists a prime ideal $\mathfrak{p}_{o}$ contained in $\mathfrak{p}$ such that $\operatorname{dim}\left(A / \mathfrak{p}_{o}\right)_{\mathfrak{p}} \geq p(A)-\operatorname{dim} A / \mathfrak{p}$ and $A_{\mathfrak{p}_{o}}$ is non-Cohen-Macaulay. In fact, let $\mathfrak{a}(A)=\mathfrak{a}_{o}(A) \ldots \mathfrak{a}_{d-1}(A)$, where $\mathfrak{a}_{i}(A)$ is the annihilator of $H_{\mathfrak{q}}^{i}(A)$ and $d=\operatorname{dim} A$. Since $A$ is equidimensional and catenary, we have $\operatorname{nCM}(A)=V(\mathfrak{a}(A))$ by $[\mathrm{Sch}], 2.4 .6$. Moreover, since $\operatorname{nCM}(A)$ is equidimensional, there exists a prime ideal $\mathfrak{p}_{o}$ containing $\mathfrak{a}(A)$ such that $\mathfrak{p}_{o} \subseteq \mathfrak{p}$ and $\operatorname{dim} A / \mathfrak{p}_{o}=\operatorname{dim} A / \mathfrak{a}(A)$. Therefore $A_{\mathfrak{p}_{o}}$ is non-Cohen-Macaulay, and $\operatorname{dim} A / \mathfrak{p}_{o}=p(A)$ by $(2.2)$. Then $\operatorname{dim}\left(A / \mathfrak{p}_{o}\right)_{\mathfrak{p}}=$ $\operatorname{dim} A / \mathfrak{p}_{o}-\operatorname{dim} A / \mathfrak{p}=p(A)-\operatorname{dim} A / \mathfrak{p}$, as required.

(ii) $\Rightarrow$ (i). Let $Z$ be an abitrary irreducible component of $\operatorname{nCM}(X)$ with the unique generic point $z$. As in the proof of (i) $\Rightarrow$ (ii) we have $d p(z)=\operatorname{dim} O_{X, z}$. Therefore $\mathrm{nCM}(X)$ is equicodimensinal since $d p$ is a constant function on $\mathrm{nCM}(X)$. Suppose now that $\operatorname{nCM}(X)$ is not equidimensional. Then, as $X$ is locally equidimensional and catenary, there exists a point $x \in \mathrm{nCM}(X)$ such that $O_{X, x} / J_{x}$ is not an equidimensional local ring, where $J$ is a sheaf of ideals defining $\mathrm{nCM}(X)$. Put $O_{X, x}=A, J_{x}=I \subseteq A$. Let $\mathfrak{m}$ the maximal ideal of $A$. Since $A / I$ is not equidimensional, there exists a prime ideal $\mathfrak{p} \in \operatorname{Ass}(A / I)$ such that $\operatorname{dim} A / \mathfrak{p}<\operatorname{dim} A / I$ and $p(\mathfrak{p})=0$. Therefore, $d p(\mathfrak{p})=\operatorname{dim} A_{\mathfrak{p}}-p(\mathfrak{p})=\operatorname{dim} A_{\mathfrak{p}}=\operatorname{dim} A-\operatorname{dim}(A / \mathfrak{p})>$ 
$\operatorname{dim} A-\operatorname{dim}(A / I)=\operatorname{dim} A-p(A)=d p(\mathfrak{m})$ by $(2.2)$, (ii). Thus the function $d p$ is not a constant on $\mathrm{nCM}(X)$. The theorem is proved.

(3.5) Remarks. The following example shows that Theorem (3.4) does not hold without the assumption of the equidimensionality of $X$. Let $X_{1}, \ldots, X_{5}$ be indeterminates over an algebraic closed field $k$. We put $R=k\left[X_{1}, \ldots, X_{5}\right], \mathfrak{p}_{1}=$ $\left(X_{1}, X_{2}, X_{3}, X_{4}\right) R, \mathfrak{p}_{2}=\left(X_{1}, X_{2}, X_{4}, X_{5}\right) R$ and $\mathfrak{p}_{3}=\left(X_{1}, X_{3}, X_{4}, X_{5}\right) R$. We consider a ring $A$ defined by $A=S^{-1}(R / \mathfrak{a})$, where $\mathfrak{a}=\left(X_{1}, X_{2}\right) R \cap\left(X_{3}, X_{4}\right) R \cap$ $\left(X_{1}, X_{4}, X_{5}\right) R$ and $S=R \backslash\left(\mathfrak{p}_{1} \cup \mathfrak{p}_{2} \cup \mathfrak{p}_{3}\right)$. Then, $X=\operatorname{Spec}(A)$ is not equidimensional. It is not difficult to check that $\operatorname{nCM}(X)=\left\{\mathfrak{p}_{1} A, \mathfrak{p}_{2} A, \mathfrak{p}_{3} A\right\}$; hence $\operatorname{nCM}(X)$ is biequidimensional. On the other hand, we can easily show that $p\left(A_{\mathfrak{p}_{1}}\right)=0$ and $p\left(A_{\mathfrak{p}_{2}}\right)=p\left(A_{\mathfrak{p}_{3}}\right)=1$. Therefore $d p\left(A_{\mathfrak{p}_{2}}\right)=d p\left(A_{\mathfrak{p}_{3}}\right)=1 \neq 2=d p\left(A_{\mathfrak{p}_{1}}\right)$; so $d p$ is not constant on $\mathrm{nCM}(X)$.

However, we do not have any example which shows the necessary of the equidimensionality of $X$ in Theorem (3.2). Thus we have the following question, whose answer we still do not know:

Question. Let $X$ be a noetherian scheme admitting a dualizing complex. Is the function $d p: X \longrightarrow \mathbb{Z}$ semicontinuous ?

\section{ACKNOWLEDGMENT}

The author would like to thank the referee for pointing out some mistakes in the original manuscript.

\section{REFERENCES}

[C1] Cuong, N.T., On the dimension of the non-Cohen-Macaulay locus of local rings admitting dualizing complexes, Math. Proc. Cambridge Phil. Soc. 109(2) (1991), 479-488. MR 92b:13034

[C2] Cuong, N.T., On the least degree of polynomials bounding above the differences between lengths and multiplicities of certain systems of parameters in local rings, Nagoya Math. J. 125 (1992), 105-114. MR 93c:1348

[C-M] Cuong, N.T., Minh, N.D., On the openness of the locus of points having polynomial type bounded above by a constant, Vietnam J. of Math. 20(1) (1992), 71-78. MR 96j:13023

[G] Grothendieck, A., Elements de Geometrie Algebrique, IV, vol. 24, Publ. Math. I.H.E.S., 1965. MR 33:7330

[H1] Hartshorne, R., Residues and Duality, vol. 20, Lect. Notes Math. Berlin Heidelberg New York, Springer-Verlag, 1966. MR 36:5145

[H2] Hartshorne, R., Algebraic Geometry, Berlin Heidelberg New York, SpringerVerlag, 1977. MR 57:3116

[M] Matsumura, H., Commutative Algebra, Second Edition, London, Benjamin, 1980. MR 82i: 13003

[S] Serre, J-P., Algèbre locale. Multiplicités, vol. 11, Lect. Notes Math. Berlin Heidelbeg New York, Springer-Verlag, 1965. MR 34:1352

[Sch] Schenzel, P., Dualisierende Komplexe in der lokalen Algebra und Buchsbaum-Ringe, vol. 907, Lect. Notes Math. Berlin Heidelberg New York, Springer-Verlag, 1982. MR 83i: 13013

Institute of Mathematics, P.O. Box 631, BoHo, 10.000 Hanoi, Vietnam

E-mail address: ntcuong@thevinh.ac.vn 